\title{
Strategy for Revising and Updating Maintenance of Certification Self-Assessment Modules (SAMs)
}

\author{
Michael D. Hagen, MD, Martin A. Quan, MD, Roger Fain, MA, \\ and William DeBord, MSLS
}

In 2004 the first Self-Assessment Modules (SAMs) went online as a resource for fulfilling the part II requirement of Maintenance of Certification for Family Physicians. As time has gone by, Diplomates have occasionally asked about the process the American Board of Family Medicine uses to revise and update the content of the SAMs. Given the pace of change in medical knowledge, American Board of Family Medicine staff have found it necessary to use multiple strategies to keep the SAMs current.

In an effort to obtain automated summaries of knowledge updates, our information specialist has worked with a physician to identify sets of search terms, called hedges, for use in the MedLINE/ PubMed My NCBI tool available at the National Library of Medicine's PubMed website (https://www. ncbi.nlm.nih.gov/account/?back_url=http $\% 3 \mathrm{~A} \% 2 \mathrm{~F} \%$ 2Fwww.ncbi.nlm.nih.gov\%2Fpubmed $\% 2 \mathrm{~F}$ ). This site allows users to create and store literature search strategies that run on a periodic basis to retrieve recent information on a given topic. These searches have been developed for every SAM item, and the staff reviews the results on a regular basis to identify significant new content or changes to existing information.

A second strategy is to have a member of the knowledge team that developed the SAM review the complete set of questions. Those that are no longer accurate or current are modified or replaced accordingly. In addition, some questions are re-

Conflict of interest: Dr. Hagen, Mr. DeBord, and Mr. Fain are employed by the ABFM.

Corresponding author: Michael D. Hagen, MD, 1648 McGrathiana Parkway, Suite 550, Lexington, KY 40511 (E-mail: hagen@theabfm.org). moved to make room for new ones that reflect important developments.

Since Diplomates are permitted to repeat a SAM for Maintenance of Certification for Family Physician credits after 5 years have lapsed, a more in-depth review of the SAM is performed every 5 years by members of the original development team, as well as by new additions to the group, with a goal of replacing at least a third of the questions.

Finally, the commenting feature accessible to Diplomates after completing the SAM questions has proven to be a helpful tool for keeping SAMs up to date. As with the hedges, staff members review this feedback on a regular basis. In addition to providing input regarding ambiguous or controversial content, these comments can also alert our staff to content that has become problematic because of new recommendations or studies.

After a physician has revised or replaced a question, the changes go to the content development department. The new or revised content is then edited and incorporated into the department's database, where the questions are stored and maintained. From there, the new content is exported to the information technology department, where it is moved into another database. The editors then review the questions once more in the format used on the web, and once they are approved the changes are deployed to our website.

While complex and labor intensive, these strategies have served to keep the SAMs current and assure our Diplomates that the content remains cutting edge and dynamically responsive to the changing field of family medicine. 